\title{
IL Messaggio di RAMón GAYA ${ }^{1}$
}

LeOnardo CAmmarano

Pintor, escritor y traductor de Ramón Gaya

Prima di trasferirmi in Francia, negli anni '70, ebbi la fortuna d'incontrare spesso Ramón Gaya, e di tanto in tanto il privilegio di seguirlo con la mia casetta di acquarelli (che era un suo regalo, tra l'altro) nelle sue peregrinazioni pittoriche nella vecchia Roma o lungo l'Appia Antica. E qualche volta anche a Napoli, nei bellissimi dintorni delle ville vesuviane, quando per un certo tempo si pensò di affidare alle sorelle Zambrano la «Ginestra» di Antonio Ranieri e di Giacomo Leopardi. Di quella bellezza, già allora deturpata dalla speculazione edilizia, ricordo che Ramón diceva: «È incredibile quanto sia resistente!».

La sua sola presenza bastava ad inibire le scialbe frivolezze della vuota conversazione quotidiana. Della sua conversazione colpiva invece la qualità, che testimoniava di uno spirito costantemente rivolto all'essenziale. Il suo interesse unico era il carattere (dunque l'essere, avrebbe detto Hegel) di cose e persone. Questo suo modo di porsi, una sorta di gentile intransigenza, emerge assai chiaro anche nei suoi scritti: fedele alla sua natura di artista, discutendo, parlando, insomma: vivendo, continuava a restare in sintonia «con le cose stesse», in perenne colloquio con quella che Robert Musil chiama «la realtà spettrale».

Per adottare questo atteggiamento, che Benedetto Croce avrebbe definito di «disinteressato interesse», occorre una qualità umana che purtroppo non è facile trovare; sarebbe un atteggiamento oggi «anticonformistico» e pertanto assai utile. Come prevedeva Ortega, siamo diventati tutti una obbediente massa damnationis. Se il «primo» Wittgenstein, quasi inaugurando questa attuale insipienza artisticofilosofica, sosteneva che «di ciò che non si riesce a dire, bisogna tacere», ebbene l'opera di Ramón Gaya, dipinta, scritta o soltanto «parlata», sostiene il perfetto contrario: è «proprio di ciò che non si riesce a dire che bisogna parlare». È questa la modernità di Gaya: egli..., disobbedendo a Wittgenstein, rifiutava di dire «ciò di cui si può dire», ovvero le solite banalità.

Si valuta subito l'importanza di un simile stato d'animo se si pensa che esso lo faceva integralmente estraneo alle problematiche psicologiche che solitamente

\footnotetext{
${ }^{1}$ Texto inédito, fechado el 2 de octubre de 2010, por cortesía de Isabel Verdejo.
} 
sogliono assillare noi uomini con disgustosa iterazione. Egli offriva lo spettacolo di un uomo deciso ad ignorarsi. Donde il tono raffinato delle sue tele, dei suoi scritti, della sua conversazione. Integralmente libero dai patetismi della «autobiografia», costituiva la prova vivente di quanto afferma Adorno contra tutti gli attuali teorici di estetica: l'estetica non sa che farsi della freudiana «sublimazione». Il che significa dire, come telegraficamente precisa J. F. Revel: l'opera d'arte non è un «Rorschach».

Questa non-lagrimosità è una nota spesso presente, se non addirittura prevalente, in quel grandioso «pezzo d'Europa» che è la Spagna: miniera di diversità nella quale, nel nostro tempo plebeo, sarebbe importante più spesso inoltrarsi. Il famoso «realismo» ispanico è nei fatti un luminoso dualismo: un realismo convinto del fatto che occorre perseguire la realità, certo, $m a$ solo perché essa è l'involucro di segreti messaggi. Persino il vitalismo di Ortega da immanentismo assoluto in accordo col le vedute di von Uexküll («io sono un Io + le mie circostanze»), si trasformò prima in prospettivismo e finalmente, grazie alla «crisi» europea da lui stesso scoperta, in esigenza di autotrascendimento.

Autotrascendimento? Vocazione?... Imbrattati dalla televisione e dal «politicismo», sopravviviamo abbarbicati alla convinzione opposta: è di moda la vanità dell'Io considerato come segue: «sono quel che sono»... Visto come fine a sé stesso e dunque privo di contenuto, 1'Io non è che pesante materia. Materia, in noi e nelle cose: non ci si occupa d'altro. Di qui l'estetica piccoloborghese: arte come espressione... della propria personalità (quasi fosse facile averne una, di «personalità»!), come autoterapia (Herbert Read e la già ricordata sublimazione), come documento sociologico o addirittura politico (i «manifesti»; la «provocazione»)... Insomma: arte al costante servizio del nostro ipertrofico Io.

Ben lungi da questo moi hä̈ssable, Ramón Gaya persegue la realtà «concava» dello Spirito. Il «vuoto» tanto sottolineato nelle sue teorizzazioni di estetica, corrisponde infatti al limpido stato di un'anima svincolatasi dall'Io e dalle sue ancillari problematiche.

Esempi e conferme, in ambito ispanico, si affollano alla mente: il sarcasmo di Cervantes (e la sua «confessione» all'inizio del Viaje al Parnaso); lo stupefacente «vuoto» delle Meninas; il silenzio dei bodegones di Zurbarán, la risoluta affermazione di Unamuno del carattere acheiropoietico della grande arte, la poesia kenotica di san Govanni della Croce, che giunge a Dio dileguandosi...: nostrum agere est pati Deum in nobis operantem. E così anche nella «inteligencia sentiente» di Xavier Zubiri, secondo il quale nel processo della conoscenza è la realtà che si accosta al meditante che se ne fa degno, non viceversa. E non è un semplice caso se, ancora in ambito culturale ispanico, María Zambrano sostiene, contro l'autorevole parere di Mircea Eliade, che il «fatto primo» che segnò l'apparizione dell'Uomo fu la «creazione 
di un vuoto» nella ressa della realtà: fu quest'ultima, non la «leggera» solitudine, il nemico primordiale da abbattere. Teoria fondativa talmente pregnante, che si potrebbe elevarla a divisa generale della nostra umana avventura.

Gaya è un pittorico conseguimento di questo modo dell'umano. Si può citare a conferma di ciò la sua significativa preferenza per il miraggio e le sue leggi: come nei «concavi» paesaggi, veneziani, fiorentini, romani, nei quali le cose si fanno presenti dileguando; o nei suoi «omaggi», che alludono alle opere d'arte da lui amate e prescelte facendone echi leggeri di spirituali «presenze»; o nel carattere etereo, di prezioso e vibratile «riflesso», dei suoi «interni», dei suoi fiori, dei suoi cristalli, dei suoi vetri semitrasparenti, che tutti alludono al sempre presente, ma spesso inavvertito, mistero della creazione. Vuoto: sì. Ma l'unico vuoto da noi uomini «tecnicamente» attingibile è appunto il vuoto dall'Io.

Una traiettoria artistica che conferma, contro l'andazzo dei tempi, che l'Io è mezzo, non mai fine. Un segreto che consiste nel farsi presenti certo, ma... per farsi da parte; un atteggiamento che, considerato dall'altro lato, ovvero dal punto di vista psicologico, si rivela una forma di aristocratico coraggio. 